\title{
Electrolyte imbalance in critically ill paediatric patients
}

\author{
Faizia Naseem ${ }^{1}$, Ayesha Saleem ${ }^{2}$, \\ Imtiaz Ahmed Mahar ${ }^{3}$, Fehmina Arif ${ }^{4}$
}

\begin{abstract}
Objective: To determine the frequency and outcome of electrolyte imbalance in seriously ill children admitted in Paediatric Intensive Care Unit (PICU) of a public sector hospital in Karachi.

Methods: All children between the ages of one month to 12 years admitted in PICU from May 2017 to October 2017 were included. Blood samples were drawn to determine the baseline sodium, potassium, calcium, magnesium and phosphorous and followed 24 hourly or earlier, if needed (Those who had imbalance at admission or on subsequent repeat labs as per protocol).

Results: A total of 101 children were included in the study. Electrolyte imbalance was seen in $84 \%$. A single electrolyte imbalance was noted in $30.58 \%$. Hypocalcemia was the most frequent abnormality noted in $57.6 \%$. Among the total expiries during the study period $91 \%$ had electrolyte imbalance. Mortality within 48 hours and duration of stay was also increased in these patients.
\end{abstract}

Conclusion: Electrolyte imbalance is an important prognostic indicator in critically ill patients.

KEYWORDS: Critically ill children, Electrolyte imbalances, Morbidity, Mortality.

doi: https://doi.org/10.12669/pjms.35.4.286

How to cite this:

Naseem F, Saleem A, Mahar IA, Arif F. Electrolyte imbalance in critically ill paediatric patients. Pak J Med Sci. 2019;35(4):1093-1098. doi: https://doi.org/10.12669/pjms.35.4.286

This is an Open Access article distributed under the terms of the Creative Commons Attribution License (http://creativecommons.org/licenses/by/3.0), which permits unrestricted use, distribution, and reproduction in any medium, provided the original work is properly cited.

\section{INTRODUCTION}

Electrolyte imbalances are not uncommon in critically ill paediatric patients. ${ }^{1}$ When present; they can significantly affect the outcome of

1. Faizia Naseem, MBBS, FCPS.

Assistant Professor

2. Ayesha Saleem, MBBS, FCPS.

Assistant Professor, Paediatric Unit III,

DUHS, Karachi, Pakistan.

3. Imtiaz Ahmed Mahar, MBBS, DCH.

Senior MO, Dr. Ruth KM Pfau Civil Hospital,

Karachi, Pakistan.

4. Prof. Fehmina Arif, MBBS, FCPS.

1,4: Paediatric Unit-I,

DUHS, Karachi, Pakistan.

Correspondence:

Faizia Naseem, MBBS, FCPS.

Assistant Professor

Paediatric Unit-I,

DUHS, Karachi, Pakistan.

Email: faizianasim@yahoo.com

* Received for Publication:

December 31, 2018

* Revision Received:

* Revision Accepted:
June 1, 2019

June 15, 2019 patients. Critical care provision through paediatric intensive care units (PICU) is aimed at maintaining 'homeostasis' in the body which is vital for the organ's support and optimal function. This involves not only fluids but also electrolytes balance. ${ }^{2}$

Major electrolytes important in this regard are sodium, potassium, calcium, magnesium and phosphorus. ${ }^{3}$ Their imbalance in either direction i.e. lower or higher than normal values can affect cellular processes, which can significantly affect morbidity and mortality. ${ }^{4}$ These imbalances also result in longer stay in hospitals, ${ }^{5}$ thus adding significantly to the costs of management. Thus early recognition and intervention to correct these imbalances is essential to avoid poor outcome. ${ }^{6}$

Five possible mechanisms for the occurrence of electrolyte imbalance are the underlying disease process, end organ injury, fluid and electrolyte interventions, use of medications with potential of electrolyte derangements and application of critical care technology i.e. positive pressure ventilation. $^{7}$ 
There is a lot of published adult data addressing this problem but paediatric data has just begun to generate especially over the last decade with the growth of paediatric critical care medicine subspecialty. This study was conducted to identify the magnitude and various aspects of the problem in Pakistani children admitted in Paediatric intensive care unit of a public hospital.

\section{METHODS}

This was an observational study conducted over six months from May 2017 to October 2017, in Dr. Ruth KM Pfau Civil Hospital Karachi after approval from IRB (Institutional Review Board). Children admitted in PICU during the study period aged one month and older up to 12 years of age, were enrolled after written consent from their parents. Children with history of using electrolyte solutions prior to admission were excluded. All the enrolled patients were followed from admission till their stay in PICU. They were regularly monitored for the presence or later development of electrolyte imbalance. A detailed history and thorough physical examination was done. The demographic data of all the participants regarding age, sex and admitting diagnosis was noted.

Blood samples were drawn on admission to find out the initial serum levels of sodium, potassium, calcium, magnesium and phosphorus. There after the levels were repeated every twenty four hourly or earlier (if needed). Any value below or above the following cut off values was considered abnormal indicating electrolyte imbalance. ${ }^{8}$

Sodium: $135-145 \mathrm{mg} / \mathrm{dl}$

Potassium: one month to five month: $3.5-5.6 \mathrm{mg} / \mathrm{dl}$

Six month to one year: $3.6-6.1 \mathrm{mg} / \mathrm{dl}$

$>1$ year: $3.3-4.6 \mathrm{mg} / \mathrm{dl}$

Calcium (Total): child: $8.8-10.8 \mathrm{mg} / \mathrm{dl}$,

Thereafter 8.4-10.2 mg/dl,

(Ionized calcium): $4.5-5.6 \mathrm{mg} / \mathrm{dl}$.

Phosphorus: 1-3 year: $3.8-6.5 \mathrm{mg} / \mathrm{dl}$

4-11 year: $3.7-5.6 \mathrm{mg} / \mathrm{dl}$

$>12$ year: $2.9-5.4 \mathrm{mg} / \mathrm{dl}$

Magnesium: up to 2 year: $1.6-2.6 \mathrm{mg} / \mathrm{dl}$

2-14 year: $1.5-2.3 \mathrm{mg} / \mathrm{dl}$

Types of intravenous fluid given to patient, use of diuretics, steroids, inotropes and positive pressure ventilation were noted. Complications during stay like development of acute kidney injury (AKI), multi organ dysfunction syndrome (MODS), congestive cardiac failure $(\mathrm{CCF})$, and syndrome of inappropriate secretion of ADH (SIADH) were noted. The final outcome i.e. discharges or expiry along with length of stay in PICU was also noted.

Data were analyzed using SPSS version 16. Descriptive statistics were applied to describe the results in terms of percentages and frequencies. Chi square was applied for association of electrolyte imbalance with the outcome, $\mathrm{P}$-value $<0.05$ was considered significant.

\section{RESULTS}

During the study period, 101 children admitted in PICU were enrolled for the analysis. Among these children, $61(60.39 \%)$ were male and 40 (39.60\%) were female. Seventy five $(74.25 \%)$ were under five years of age, $17(16.8 \%)$ between 5 and 10 years of age and 9 (8.91) were above 10 years of age.

Regarding the admitting diagnosis, majority i.e. $31(30.69 \%)$ had respiratory illness, $26(25.7 \%)$ had neurological illness, $18(19.80 \%)$ has sepsis/ infection, 12 (11.88\%) had cardiovascular problem, 4 $(3.96 \%)$ had gastrointestinal ailment and 10 (9.90\%) were categorized in miscellaneous group including endocrine diseases.

Table-I: Descriptive characteristics of study population $(\mathrm{N}=101)$.

\begin{tabular}{|c|c|c|}
\hline Characteristics & No & $\%$ \\
\hline \multicolumn{3}{|l|}{ Sex: } \\
\hline Male & 61 & 60.39 \\
\hline Female & 40 & 39.60 \\
\hline \multicolumn{3}{|l|}{ Age groups: } \\
\hline$<5$ years & 75 & 74.25 \\
\hline $5-10$ years & 17 & 16.83 \\
\hline$>10$ years & 09 & 8.91 \\
\hline \multicolumn{3}{|l|}{ Electrolyte Imbalance: } \\
\hline -Present & 85 & 84.15 \\
\hline Absent & 16 & 15.84 \\
\hline \multicolumn{3}{|l|}{ Outcome: } \\
\hline $\begin{array}{l}\text {-Discharged } \\
\text { (including } 2 \text { LAMA and } 2 \text { referrals) }\end{array}$ & 78 & 77.22 \\
\hline -Expired & 23 & 22.77 \\
\hline \multicolumn{3}{|l|}{ Disease Distribution: } \\
\hline -Respiratory & 31 & 30.69 \\
\hline -CNS & 26 & 25.74 \\
\hline -Infectons/Sepsis & 18 & 17.82 \\
\hline -CVS & 12 & 11.88 \\
\hline -GIT & 04 & 3.96 \\
\hline -Others & 10 & 9.90 \\
\hline \multicolumn{3}{|l|}{ Length of stay in PICU: } \\
\hline$<48$ hours & 42 & 41.58 \\
\hline 48 hours- 4 days & 27 & 26.73 \\
\hline 5 days- 9 days & 19 & 18.81 \\
\hline$>10$ days & 13 & 12.87 \\
\hline
\end{tabular}


Table-II: Pattern of electrolyte imbalance $(\mathrm{n}=85)$.

\begin{tabular}{lcc}
\hline Electrolyte & No & $\%$ \\
\hline 1. Sodium: & 32 & 37.64 \\
Hypernatremia & 20 & 23.52 \\
Hyponatremia & & \\
2. Potassium: & 16 & 18.82 \\
Hyperkalemia & 26 & 30.58 \\
Hypokalemia & & \\
3. Calcium: & 0 & 0 \\
Hypercalcemia & 49 & 57.64 \\
Hypocalcemia & & \\
4. Magnesium: & 18 & 21.17 \\
Hypermagnesemia & 06 & 7.05 \\
Hypomagnesemia & & \\
5. Phosphorus: & 10 & 11.76 \\
Hyperphosphatemia & 30 & 35.29 \\
Hypophosphatemia & 26 & 30.58 \\
Imbalance of single electrolyte & 22 & 25.88 \\
Imbalance of two electrolytes & 20 & 23.52 \\
Imbalance of three electrolytes & 11 & 12.94 \\
Imbalance of four electrolytes & 06 & 7.05 \\
Imbalance of five electrolytes & 80 & 94.11 \\
Imbalance on admission & 05 & 5.88 \\
Imbalance developed later & & \\
\hline
\end{tabular}

Electrolyte imbalance was noted in 85 (84.15\%) children. It was present in $80(94.11 \%)$ at the time of admission. Development of imbalance later during the stay was noted in $25(29.41 \%)$ including $5(5.88 \%)$ cases free of imbalance at admission (Table-I). Majority of them i.e. $26(30.58 \%)$ had imbalance of a single electrolyte. However; $22(25.88 \%)$ had imbalance of two electrlytes, $20(23.52 \%)$ had imbalance of three electrolytes, $11(12.94 \%)$ had imbalance of four electrolytes and $6(7.05 \%)$ had imbalance of all five electrolytes (Table-I).

The most frequently noted abnormality was hypocalcemia seen in $49(57.64 \%)$ patients (TableII). Hypernatremia was seen in $32(37.64 \%)$ cases and hypophosphatemia was present in 30 $(35.29 \%)$, Hypokalemia was noted in $26(30.58 \%)$ and hypermagnesemia in $18(21.17 \%)$ (Table-II). Regarding use of medications having potential for electrolyte imbalances, $75(74.25 \%)$ required inotropes for stabilization, $61(60.39 \%)$ required steroids and $44(43.56 \%)$ needed diuretics. Mechanical ventilation was required in 56 $(55.44 \%)$ children (Table-III). In-depth analysis of patients having electrolyte imbalance revealed that morbidity and mortality were both increased in such cases i.e. all AKI (Acute kidney injury), MODS (Multiorgan dysfunction syndrome), CCF (Congestive cardiac failure) and SIADH (Syndrome of inappropriate secretion of $\mathrm{ADH}$ ) cases had electrolyte imbalance.

Out of these 101 admissions, total discharges from PICU were 78 (including 2 LAMA and two referrals) and there were 23 expiries. Out of the total

Table-III: Morbidity profile and length of stay in PICU among patients with and without electrolyte imbalance.

\begin{tabular}{lccc}
\hline Characteristics & $\begin{array}{c}\text { With electrolyte } \\
\text { imbalance }(n=85)\end{array}$ & $\begin{array}{c}\text { Without electrolyte } \\
\text { imbalance }(n=16)\end{array}$ & P-value \\
\hline Morbidity & & & \\
a. Required level of care:- & & & 0.08 \\
Need for ventilator (56) & $46(82.14 \%)$ & $10(17.85 \%)$ & 0.05 \\
Need for inotropes (66) & $57(86.36 \%)$ & $09(13.63 \%)$ & 0.08 \\
Need for diuretics (44) & $40(90.90 \%)$ & $04(9.09 \%)$ & 0.08 \\
Need for steroids (61) & $50(81.96 \%)$ & $11(18.03 \%)$ & 0.29 \\
b. Complications:- & & & 0.25 \\
AKI & $10(11.76 \%)$ & 0 & 0.29 \\
MODS & $11(12.94 \%)$ & 0 & 0.79 \\
CCF & $10(11.76 \%)$ & 0 & 0.062 \\
SIADH & $02(2.35 \%)$ & $10(62.50 \%)$ & 0.42 \\
Length of Stay:- & & $3(18.75 \%)$ & 0.54 \\
$<$ 48 hours & $32(37.64 \%)$ & $2(12.50 \%)$ & 0.54 \\
48 hours-4 days & $24(28.23 \%)$ & $1(6.25 \%)$ & $<0.001$ \\
5 days-9 days & $17(20.00 \%)$ & & \\
$>10$ days & $12(14.11 \%)$ & $14(87.50 \%)$ & \\
Discharged:- & & 0 & \\
(Including 2 LAMA and 2 referrals) & $64(75.29 \%)$ & &
\end{tabular}


Faizia Naseem et al.

Table-IV: Mortality profile among patients with and without electrolyte imbalance.

\begin{tabular}{lccc}
\hline Characteristics & $\begin{array}{c}\text { With electrolyte } \\
\text { imbalance }(n=85)\end{array}$ & $\begin{array}{c}\text { Without electrolyte } \\
\text { imbalance }(n=16)\end{array}$ & $\begin{array}{c}\text { P-value } \\
\text { Expiries (23) }\end{array}$ \\
Within 48 hours (14) & 21 & 02 & $<0.001$ \\
-b/w 48 hours-4days & 12 & 02 & 0.001 \\
-b/w 5days-9days & 4 & 0 & 0.001 \\
>10 days & 3 & 0 & 0.005 \\
With imbalance of:- & 2 & 0 & $<0.001$ \\
Up to two electrolytes & & 0 & $<$ \\
Three or more electrolytes & 7 & 0 & $<0.001$ \\
With imbalance seen:- & 14 & & 0 \\
On admission & 18 & 0 & \\
During stay & 3 & & \\
\hline
\end{tabular}

expiries during the study period, 21 (91.30\%) had electrolyte imbalance, making it a significant risk factor for mortality.

Electrolyte imbalance was also significantly seen $(85.71 \%)$ in 12 out of 14 deaths in $1^{\text {st }} 48$ hours of PICU stay. However the length of PICU stay was increased in each category of children with electrolyte imbalance especially beyond 48 hours.

\section{DISCUSSION}

Among 101 enrolled children, overall electrolyte abnormalities were present in $85 \%$ (84.15\%) of the cases. Since the five electrolytes were considered together and imbalance among any of them was noted, this might be the reason for the high incidence. Most of previous studies focused on only one or two electrolytes. Rao and Thomas, found the incidence to be $32 \%$, but they focused only on sodium and potassium. ${ }^{1}$ Panda and Save mentioned $44.3 \% .^{5}$

Cummings BM found potassium abnormalities alone to be around 40\%. ${ }^{9}$ Study done by Agarwal $\mathrm{N}$, looking at all five electrolytes showed 60\% incidence. ${ }^{3}$ This proves the statement (and rather modifies it) that electrolyte abnormalities are very common in critically ill children.

Majority had respiratory $(30.69 \%)$, neurological $(25.74 \%)$ and infective/sepsis $(19.80 \%)$ etiology as noted in previous studies.3,10,11 The reason for less number of gastrointestinal cases $(3.96 \%)$ could be the initial stabilization in ER and later shifting to ward upon improvement thus bypassing the PICU stay. This is important because gastroenteritis in children is the major source of electrolyte imbalance, but prompt and proper treatment may avoid PICU admission.
Although majority had abnormality of a single electrolyte, mixed disorders with combination of two, three, four and all five electrolytes were also seen (Table-II). Majority i.e. 80 (79.20\%) had the abnormalities at the time of admission, 20 of them developed additional imbalances during stay. While five patients who were free of imbalance at admission developed them later, indicating the possible mechanism pointed earlier. Regarding the patterns of electrolyte imbalance, hypocalcemia was the most frequent abnormality noted in 49 (57.4\%) cases (Table-II). Previous studies have mentioned the incidence of hypocalcemia in critically ill children to be around $40 \%{ }^{3}$ and $47.5 \% .{ }^{12}$ One possible explanation for such a high incidence of hypocalcemia is the high prevalence of Vitamin D deficiency in Pakistani children up to $77 \% .^{13}$

The presence of dysnatremias (either hypo or hypernatremia) in intensive care unit has been reported to be around $30 \%{ }^{14,15}$ Most of the literature has reported hyponatremia to be more prevalent than hypernatremia i.e. $23.2 \%$ vs. $16.7 \%{ }^{16}$, $27.43 \%$ vs. $3.5 \% 5,50.5 \%$ vs. $9.4 \% .{ }^{3}$ Sachdev A noted hyponatremia to be $19.3 \% .^{11}$

However, we found hypernatremia in $37.64 \%$ cases and hyponatremia in $23.52 \%$ cases. This might be due to the institutional policy of maintenance intravenous fluid to be the $0.9 \%$ saline rather than half strength or other hypotonic solutions in children above one month of age.

Hypokalemia was observed in $30.58 \%$ cases and hyperkalemia in $18.82 \%$ cases. Previous studies have mentioned the incidence of hypokalemia as $40 \%{ }^{9}, 34.4 \%^{3}$ and $22.1 \%{ }^{16}$ and hyperkalemia as $11.2 \%^{16}, 16.12 \%^{3}$ and 29\%. ${ }^{9}$ Hypophosphatemia was noted in $35.29 \%$ cases. This is similar to the 
incidence reported by Antachopoulos as $37.5 \% .^{17}$ Although ME Santana found the incidence up to $61 \%$ in critically ill children. ${ }^{18}$

As far as magnesium is concerned, we found more cases of hypermagnesemia up to $21 \%$ than hypomagnesemia in $7 \%$ (Table-II). This is in contrast to most of the published data showing hypomagnesemia to be more common than hypermagnesemia., ${ }^{2,3}$ In 17 out of 18 cases, it was seen as a part of mixed electrolyte disorders. Isolated hypermagnesemia was seen in a single case only.

Morbidity was significant in cases of electrolyte imbalance both in terms of required level of care and emergence of complications. Ventilatory support was required in $82 \%$ of these children. Similarly diuretics were required in $90.90 \%$, inotropes were required in $88 \%$ and steroids were needed in $81.96 \%$, indicating the need for high level of care (Table-III).

It is claimed that medications commonly used in intensive care units may contribute to the electrolyte disturbances as they can interfere with the absorption of electrolytes, alter hormonal responses affecting hemostasis and can directly affect the organ function as well. Their requirement however indicates the severity of illness and they continue to be an important risk factor for later development of electrolyte imbalance. ${ }^{19}$

Morbidity in terms of complications was also significant in such cases i.e. AKI, MODS, CCF, and SIADH were seen exclusively in patients having electrolyte imbalance (Table-III). Regarding the outcome, significant number of discharges were seen in cases without electrolyte Imbalance, P-value $<0.001$ (Table-III). Overall mortality in our study was $22.77 \%(23 / 101)$, close to the mortality documented by Panda I as $23.73 \%{ }^{5}$, and by Jan M et al as $22.8 \%{ }^{20}$ (Table-IV). However, more than $90 \%$ of non survivors i.e. 21 out of 23 had electrolyte imbalance P-value $<0.01$ and among these 18 had imbalance at presentation P-value $<0.001$ (TableIV) making the presence of electrolyte imbalance at admission to be the strongest predictor of mortality because such abnormalities complicate the course of illness, irrespective of primary disease process. ${ }^{3,5}$

Mortality during first 48 hours of stay was seen in 14 cases, among which 12 (85.71\%) had electrolyte imbalance again pointing towards its significant role in poor outcome. Both single and mixed electrolyte disorders were noted and the mortality was increased in ascending order with the number of electrolyte involvement i.e. 7 of 21 non survivors had up to two abnormal electrolytes and 14 of 21 non survivors had three or more abnormal electrolytes (Table-IV).

Length of stay less than 48 hours was seen in $32(37.64 \%)$ cases of electrolyte imbalance (high early mortality) whereas it was seen in 10 $(62.50 \%)$ cases without imbalance (indicating high rates of recovery). However, the length of stay was increased in all other categories in patients with electrolyte imbalance (Table-III). This was consistent with studies conducted in the past.5,11,12 This makes electrolyte imbalance a major drain on limited health resources of poor and developing countries.

\section{CONCLUSION}

Presence of electrolyte imbalance at the time of admission is an important prognostic indicator in critically ill children irrespective of primary disease process and needs to be addressed aggressively.

Acknowledgement: We are thankful to Dow'86, Mr. Syed Arif Ali and Mrs. Batool of Research Department DUHS, for their help in statistics.

Conflict of interest: None.

Source of funding: None.

\section{REFERENCES}

1. Rao SSD, Thomas B. Electrolyte abnormalities in children admitted to pediatric intensive care unit. Indian Pediatr. 2000;37:1348-1353.

2. Balci AK, Koksal O, Kose A, Armagan E, Ozdemir F, Inal $\mathrm{T}$, et al. General characteristics of patients with electrolyte imbalance admitted to emergency department. World J Emerg Med. 2011;4(2):113-116.

3. Agarwal N, Rao Y, Saxena R, Acharya R. Profile of serum electrolytes in critically ill children: A prospective study. Indian J child Health. 2018;5(2):128-132.

4. Rukesh CC, Shalini B. Correlation between serum electrolytes and clinical outcome in children admitted to PICU. IOSR J Dent Med Sci. 2017;16:11:24-27.

5. Panda I, Save S. Study of association of mortality with electrolyte abnormalities in children admitted in pediatric intensive care unit. Int J Contemp Pediatr. 2018;5:1097-1103. doi: 10.18203/2349-3291.ijcp20181550.

6. Reddy A, Thapar RK, Gupta RK. Electrolyte disturbances in critically ill children admitted to pediatric tertiary care centre. J Evol Med Dent Sci. 2017;6:3269-3273.

7. Hauser GJ, Kulick AF. Electrolyte disorders in the PICU. In: Wheeler DS et al, eds. Pediatric critical care medicine. London: Springer-Verlag. 2014;13:147-161.

8. Greenbaum LA. Electrolyte and Acid Base Disorders. Nelson text book of Pediatrics. $20^{\text {th }}$ ed. Kliegmanetal eds. Saunder Elsevier, Philadelphia. P 346-369.

9. Cummings BM, Macklin EA, Yager PH, Sharma A, Noviski N. Potassium abnormalities in a pediatric intensive care unit: Frequency and severity. J Intensive Care Med. 2014;29(5)269-274. doi: 10.1177/0885066613491708. 
10. Haque A, Bano S. Improving outcome in pediatric intensive care unit in academic hospital in Pakistan. Pak J Med Sci. 2009;25(4):605-608.

11. Sachdev A, Pandharikar N, Gupta D, Gupta N, Gupta S, Venkatraman ST. Hospital acquired hyponatremia in pediatric intensive care unit. Indian J Crit Care Med. 2017;21:599-603. doi: 10.4103/ijccm.IjCCM_131_17.

12. Naik N, Dandge V. Role of calcium in critically ill childrenincidence of hypocalcemia in Pediatric Intensive care set up. Indian J Appl Res. 2014;4(4):409-412.

13. Fasih Z. Evaluating the Frequency of Vitamin D deficiency in the Pediatric Age Group and identifying the Biochemical Predictors Associted with Vit D Deficiency. Pediatr Ther. 2016;6:289. doi: 10.4172/2161-0665.1000289.

14. Barron R, Free Bairn R. Electrolyte disorders in the critically ill. Anaesth Intensive Care Med. 2010;11(12):523-528. doi: 10.1016/j.mpaic.2010.09.010.

15. Gibbs R, Macnaughton P. Electrolyte and metabolic disturbances in critically ill patients. Anaesth Intens Care Med. 2007;8(12):529-533. doi: 10.1016/j. mpaic.2007.09.015.

16. Elala G, Shimelis D. Patterns of electrolyte abnormalities in children 0-15 years of age admitted to pediatric Emergency and Intensive Care Units of a Tertiary Hospital. IOSR-JDMS. 2018;17(2)12-16. doi: 10.9790/0853-1702091216.

17. Antachopoulos C, Margeli A, Giannaki M, Bakoula C, Liakopoulcu T, Papassotiriou I, et al. Transient hypophosphatemia associated with acute infectious diseae in pediatric patients. Scand J Infect Dis. 2002;34:836-839.
18. Meneses ES, Fernandez JRD, Leite M, Pons H, Carvalho D, Brunow E, et al. Hypophosphatemia in critically ill children. Prevalence and associated risk factors. Pediatrcrit Care Med. 2009;10(2)234-238. doi: 10.1097/ Pcc.obo13e3181937042.

19. Buckley MS, Leblanc JM, Cawley MJ. Electrolyte disturbances associated with commonly prescribed medications in the intensive care unit. Crit Care Med. 2010;38(6):s253-s264. doi: 10.1097/CCM. Obo13e3181ddaObe.

20. Jain M, Sha A, Parajapati R. Study of electrolyte imbalance in critically ill children. Int J Int Med Res. 2015;2(2):56-59.

\section{Author's Contributions:}

FN: Data collection, interpretation, literature review and manuscript writing.

AS: Data interpretation, literature review and manuscript typing.

IAM: Data collection.

FA: Study conception and design, review and final approval of manuscript.

All authors have read and approved the final manuscript. 